Hispania Sacra, LX

121, enero-junio 2008, 131-142, ISSN: 0018-215-X

\title{
TOPOGRAFÍA MEDIEVAL DE LA CASA TOLEDANA DE LOS LASO DE LA VEGA EN LA PARROQUIA DE SAN ROMÁN*
}

\author{
POR
}

JEAN PASSINI

\begin{abstract}
RESUMEN
Los documentos medievales del Archivo del Hospital de la Misericordia favorecen la reconstitución de la evolución de una manzana, en la parroquia de san Román donde se elevaban las casas mayores que fueron sucesivamente del obispo de Coria, don Vasco Ramírez de Rivera y de la familia de los Laso de la Vega. Aunque esta casa ha sufrido varias transformaciones hasta su destrucción en el siglo XX, el autor ha podido restituirla al final del siglo XV, usando el estudio del parcelario, y situar la casa donde nació el poeta Garcilaso.
\end{abstract}

PALABRAS ClAVE: Vasco Ramírez de Rivera, Laso de la Vega, Topografía medieval, Casas medievales, Urbanismo.

\begin{abstract}
This article reveals the evolution of the medieval houses that were successively of the bishop don Vasco Ramirez de Rivera (1480-1492 and of Laso de la Vega family (1491-1616). It draws from various medieval documentary sources particularly of the Mercy's Hospital. This article examines also some actual houses of san Roma's hill. It proposes a plan of the houses at the end of the $15^{\text {th }}$ century and the situation of the native house of the poet Garcilaso
\end{abstract}

* Abreviaciones utilizadas:

A. D. P. T., Archivo de la Diputación Provincial de Toledo.

A. H. N. Archivo Histórico Nacional.

A. H. P. T., Archivo Histórico Provincial de Toledo; AMT, Archivo Municipal Toledano.

A. S. D. R., Asiento de todas las posesiones del monasterio de Santo Domingo el Real (Toledo).

I. G. N., Instituto Geográfico Nacional. 
KEY WORDS: Ramírez de Rivera, Laso de la Vega, Medieval Topography, Medieval House, Urbanism.

Recibido/Received 10-09-2006

Aceptado/Accepted 07-12-2006

La localización de la casa del poeta Garcilaso de la Vega y de su familia, en la parroquia de San Román, no está muy clara. Palazuelos ${ }^{1}$, en su guía de 1890, repite, siguiendo Sixto Ramón Parro², que se observa a la derecha de la calle que sube desde la iglesia de Santa Eulalia a Santo Domingo el Antiguo, unas grandes paredes que formaron parte de la casa de Garcilaso de la Vega, indicando que la casa grande no existe y que en su sitio se notan «unas vecindades». J. Porres $^{3}$, en la segunda mitad del siglo XX, indica que el palacio de los Laso de la Vega, conde de Arcos, constituye una «casona importante» de la calle Esteban Illán. Precisa, además, que en marzo de 1616 el conde de Arcos «vendía esta casa en la calle real, ante Juan de Soria y por 14.000 ducados, a la Compañía de Jesús», la cual la permuta con el solar del Hospital de la Misericordia. Añade, en nota citando J. Gil Calvo ${ }^{4}$, que «lindaba la casa, según escritura de la Compañía de Jesús, con otra de don Antonio Padilla (sin duda la que tiene fachada principal a la plaza apellidada por su pariente el comunero) y con casas particulares; por el otro delante había una placita «al lado de Oriente» y por la fachada cruzaba la calle Real.» La casa se transformó en hospital y pasa al final del siglo XIX a la Diputación Provincial, la cual la derribó un siglo más tarde. Últimamente, con ocasión de la rehabilitación de la Universidad de Castilla-La Mancha en 1997, el arqueólogo responsable de la excavación propone como palacio de los Laso de la Vega en la primera mitad del XVI ${ }^{5}$, toda el espacio ocupado en el siglo XIX por el Hospital de la Misericordia.

Intentaremos en este artículo reconstituir la evolución de la topografía de la manzana donde se elevaba la casa de los Laso de la Vega, en San Román, esencialmente a partir de los documentos encontrados en los Archivos del Hospital de la Misericordia. Estos documentos confirman y precisan que don Pedro Laso de la Vega Niño y Guzmán vende en 1616 los bienes de su mayorazgo en la parroquia de San Román, mayorazgo firmado por el padre del poeta don Garci Laso de la Vega y su esposa doña Sancha de Guzmán a favor de su hijo mayor, Pedro Laso,

${ }^{1}$ Palazuelos, el vizconde de, Toledo, Guía artístico-práctico, Toledo, 1890.

${ }^{2}$ S. Ramón PArro, Toledo en la mano, Toledo, 1857, reedición Toledo, 1978, 2 tomos.

3 J. Porres Martín-Cleto, Historia de las Calles de Toledo, 3 t., Toledo, 1988, Tercera edición usada aunque existe una cuarta edición revisada y aumentada en 2002. Trata de la Calle de Esteban Illán en el tomo II, p. 608, 617-630.

${ }^{4}$ J. Gil Calvo, «La iglesia de San Ildefonso y la Casa Profesa de la Compañía de Jesús en Toledo», Anales Toledanos, VI (1973) 220-221.

${ }^{5}$ A. H. P. T., sección Arqueología, J. M. RoJAs, 1997, Excavación de la Universidad de Castilla-La Mancha, plaza de Padilla.

Hispania Sacra, LX

121, enero-junio 2008, 131-142, ISSN: 0018-215-X 
en 1504, año de la compra de una casa en la colación de San Román6 ${ }^{6}$ En el documento notarial relatando esta compra se enumeran las posesiones que forman su mayorazgo, entre las cuales se mencionan «... unas casas de nuestra morada...en la dicha ciudad a la colación de san roman las quales alindan con cassas de pedro lopez de padilla y con otras de santo domingo el Real y con las calles Publicas». Primero, precisaremos la adquisición de estas casas en San Román.

\section{AdQUISICIÓN DE UNAS CASAS EN LA COLACIÓN DE SAN RoMÁN}

Como hemos mencionado anteriormente, don Garci Laso de la Vega, padre del poeta, compra, en 1504, al boticario Miguel Sierra, una casa en la colación de San Román. Esta casa se sitúa cerca del monasterio de San Clemente, al lado de las casas principales de don Garci Laso de la Vega y de su esposa doña Sancha Guzmán y linda con casa del clérigo Diego Muñoz, tributaria como la casa vendida a la superiora del monasterio de Santo Domingo el Real, Leonor Ramires de Rivera ${ }^{7}$, y con la calle Real.

Los documentos notariales o su copia que figuran en el archivo del Hospital de la Misericordia permiten seguir estos bienes desde el segundo cuarto del siglo XIV. En 1438, doña Leonor de Toledo ${ }^{8}$, segunda esposa de Mosen Ruben de Braquamonte, es propietaria de tres casas, dos en la colación de San Román, una en la colación de Santa Leocadia la Vieja (Figura 1). La casa principal, donde mora doña Leonor de Toledo, en la colación de San Román, linda con una casa de su sobrino, Fernand Álvarez de Toledo, la calle pública y la plaza que está delante de las puertas principales de esta casa. La casa con su bodega, también situada en la colación de San Román, linda de un lado con una casa de Marcos García el Viejo, del otro con una bodega de Juan Sánchez Medrano, y la calle pública. Su puerta principal da a la plaza que está delante de las puertas principales de la casa principal. La tercera casa, en la colación de Santa Leocadia la Vieja, linda de un lado con una casa de Alfonso Fernández Levi, del otro con el horno de la calle que va de las Tendillas de Sancho Bienayas al baño «que disen del Xarafy»9.

\footnotetext{
${ }^{6}$ A. D. P. T., Hospital de la Misericordia, leg. $2, \mathrm{n}^{\circ} 8$, fol. 45 v y 46.

${ }^{7}$ A. D. P. T., Hospital de la Misericordia, leg. $2, \mathrm{n}^{\circ} 8$, fol. 1 r. La casa vendida esta tributaria de 2000 mrs. y tres gallinas.

${ }^{8}$ Fernand Álvarez de Toledo, segundo señor de Valdecorneja, esposo de Leonor Fernández de Ayala, muere en 1384, tiene cinco hijos dentro de los cuales, el mayor García Alvarez de Toledo, tercero señor de Valdecorneja, Gutierre Gomez de Toledo, futuro arcediano de Toledo y Leonor de Toledo. Su nieto mayor, Fernand Álvarez de Toledo, cuarto señor de Valdecorneja, primer conde de Alba, muere en 1464 mientras su bisnieto, García Álvarez de Toledo, primer duque de Alba, muere en 1488. Ver los «Álvarez de Toledo» en J.-P. MolÉnAT, Campagnes et Monts de Tolède du XIIe au XVe siècle, Casa de Velázquez, Madrid, 1997, p. 345-348 y p. 373.

${ }_{9}^{9}$ Según nuestras investigaciones, el Baño del Zarafi se situaba debajo del Hospital del Nuncio Nuevo.
} 
Doña Leonor de Toledo da estas casas a su sobrina María de Quiñones, esposa de Alfonso de Pimentel, quien en $1462^{10}$, da estos bienes a su hijo Rodrigo de Pimentel de Benavente. El cual vende en 148011, «todas las casas mayores e menores, en la Parroquia de San Román y Santa Leocadia» a don Vasco Ramírez de Rivera, arcediano de Talavera y canónigo de Toledo ${ }^{12}$. En su testamento póstumo ${ }^{13}$, hecho por procuración en $1489^{14}$, don Vasco de Rivera deja unas casas principales en la parroquia de San Román, que lindan de una parte con la casa del cura Antonio de Contreras, tributaria al monasterio de Santo Domingo el Real, de otra con casas de Pedro López de Padilla. Deja también dos casas ${ }^{15}$ juntadas a la casa principal, que los protonotarios ponen en venta pública por 800 maravedis, con esta ocasión el 3 de julio de $1491^{16}$, el regidor Fernando Davalo compra estas casas a favor de don Garci Laso de la Vega y de doña Sancha de Guzmán. Dos meses después ${ }^{17}$, los señores don Garci Laso de la Vega y doña Sancha de Guzmán reciben del rey la autorización de comprar las otras casas que habían sido de don Vasco de Ribera, es decir, precisamente lo que fue su casa principal, abierta a una plazuela al Norte (Figura 2).

Por lo que se trata de la casa vendida por el boticario Miguel Sierra en 1504, sobre la cual la superiora del monasterio de Santo Domingo el Real tenía tributo ${ }^{18}$, está descrita en un documento de $1495^{19}$. Se sitúa frente a un ángulo del monasterio de San Clemente. Linda de un lado con la plaza delante de las casas que fueron del duque de Alba ${ }^{20}$ y son en 1495 de Pedro López de Padilla, del otro lado la casa del comendador Garci Laso de la Vega, a las espaldas «con unas casas que dissen las casas verdes que fueron del duque de Alba e después fueron del comendador Diego Carrillo», y con la calle Real.

${ }^{10}$ A. D. P. T., Hospital de la Misericordia, leg. 2, $\mathrm{n}^{\circ}$ 10, fol. 7 r, 1462.

${ }_{11}$ A. D. P. T., Hospital de la Misericordia, Lib. 8, fol. 1. Este libro reúne los documentos presentados en 1541, en la Chancillería de Valladolid por parte de don Pedro Laso de la Vega por el pleito que seguía con Alvar Gomez de Ciudad Real.

12 Don Vasco Ramírez de Rivera presidió el primer auto de fe de la Inquisición en 1485. J. Porres Martín-Cleto, «Visita guiada al Toledo de la Inquisición», Inquisición y conversos, III Curso de Cultura Hispano-Judía y Sefardí de la Universidad de Castilla-La Mancha (Toledo, 6-9 septiembre 1993), Madrid, 1994, p. 47-50.

${ }^{13}$ Don Vasco Ramírez de Ribera, nombrado obispo de Coria en el año 1486, fallece en Ocaña en 1488.

14 Ver las paginas que J. P. Molénat acuerda a la familia de los Ribera, p. 358 y siguientes, en Campagnes et Monts de Tolède du XII e au XVe siècle, Casa de Velázquez, Madrid, 1997.

${ }^{15} \mathrm{Se}$ trata de las dos casas accesorias a la casa principal, ver $\mathrm{n}^{\circ} 2$ y 3 figura 2 .

16 A. D. P. T. Misericordia, Lib. 8, fol. 17.

17 A. D. P. T. Misericordia, Lib. 81, fol. 32.

18 Doña Leonor Ramires de Ribera heredo este tributo de su padre el mariscal Payo de Ribera, A. H. N. N., Frías, C 1311, D. 11.

${ }^{19}$ A. S. D. R., 517 , fol. 315 r.

${ }^{20}$ García Álvarez de Toledo muerto en 1488 , ver nota 7. 


\section{RECAPITULACIÓN}

En 1504, don Garci Laso de la Vega, comendador de León, y doña Sancha de Guzmán tienen una casa principal, al lado de la casa de la familia de los Padilla, con su plaza principal al Norte, del lado de la calle de las Tendillas de Sancho Bienaya. Tienen también una casa accesoria, juntada a la calle que sube a San Román, con su puerta a la plaza delante de la casa principal, dos casas accesorias que abren a la calle de las Tendillas, y una casa a la esquina de la plaza delante de las casas de Padilla por un lado, de la calle que desde las Tendillas sube a San Román del otro. En $1505^{21}$ un callejón (Figura 4) conduce desde la calle de San Idelfonso a la casa principal de Garci Laso de la Vega y a una pequeña puerta de la casa de Pedro López Padilla. No se sabe más sobre la arquitectura de la casa principal, salvo de la existencia de una escalera edificada por don Vázquez de Rivera cuando rehizo su casa principal, probablemente entre 1485 y $1488^{22}$.

\section{Evolución de los bienes de los Laso de la Vega en San Román}

En $1574^{23}$, el conde de Arcos y don Antonio de Padilla obtienen del Ayuntamiento la cesión del callejón que está a las espaldas de sus casas. Unos años después, en 1591, don Pedro Laso de la Vega y Guzmán manda al Ayuntamiento una petición, en la cual escribe que en la plaza que tiene «delante de las casas principales» donde vive «se hace un muladar de todos los vecinos y moradores que viven en la vecindad de las dichas mis casas». Adjunta que aunque «se ha pregonado muchas y diversas veces por la justicia penas de dineros $y$ prisión» no se ha podido obtener el mantenimiento de la limpieza de dicha placita. Por lo cual pide que el Ayuntamiento mande sus alarifes a fin de decirle «por donde ponga puertas a la dicha plaza para que pueda cerrar de noche».

21 Santo Domingo el Real, 517: «Teresa Lopez mujer de Jerónimo del Castillo linda otra mitad de la dicha en la calle donde esta el hospital de San Ildefonso en la acera frontero del dicho hospital alinda don la dicha calle real y va a la dicha iglesia de Santa Leocadia de la otra parte alindan con otra calle que sube a la puerta pequeña de la casa de pedro lopez de padilla e que va a las casas del comendador mayor don Garcia lazo de la Vega y otra parte alinda con casa de antonio de Santa Catalina tributario a las beatas del Hospital de la misericordia en la cual mitad de casa hay...».

22 Según J. Porres, 1994, don Vasco Ramírez de Rivera hereda el mayorazgo en 1485. En el documento de 1495, anteriormente citado, se indica primero que la casa vecina de Garci Laso de La Vega fue del mariscal Payo de Ribera, quien lo ha dado a su hija Leonor de Ribera, prior del monasterio de Santo Domingo el Real. Segundo, que «antes de las heresias... solía tener libratos a la... maestre habram amori yesero» por 1.700 maravedis de tributo ${ }^{23}$. Además, precisa que en un palacio de esta casa se edificó una escalera de las casas principales de Vasco de Rivera y encima de dicho escalera se quedo una «quadra » «a censo de... capellan Diego Muñoz clerigo» por 1.000 maravedis y una gallina.

${ }^{23}$ A. D. P. T. Misericordia, Leg. 2, n¹1, fol. 22. 
Al final del mes de agosto de 1591, el Ayuntamiento acuerda que la plazuela de Laso de la Vega se conserve como está «con el pretil pegado a la calle» y que «las puertas que se han de poner se pongan en el dicho pretil».

Por otro lado, las casas accesorias de don Pedro Laso de la Vega aparecen mencionadas como linderos de cinco casas juntadas, en posesión del monasterio de Santa Clara en la parroquia del mismo, en las Tendillas de Sancho Bienayas, en un documento fechado de $1599^{24}$. Estas casas dan esquina a dos calles, una «que sube a san Roman», la otra «que baja a sancta leocadia». Así se precisa el límite Sur-Este de la manzana donde se sitúan las casas de la familia de los Laso de la Vega.

\section{RECAPITULACIÓN}

Al final del siglo XVI, don Pedro Laso de la Vega, que considera como su casa principal la casa que tiene en la parroquia de San Román, ha obtenido autorización para cerrar de noche la plaza delante de su puerta y ventanas, mediante una puerta construida sobre el pretil que sube desde la calle de las Tendillas de Sancho Bienayas. Así se confirma la existencia de una placita al Norte de la casa. La transformación de dicha plaza en un muladar, de manera habitual, por parte de sus vecinos, conduce a interrogarse sobre la ocupación efectiva de la casa por sus propietarios. Es muy probable que no ocuparan la casa de manera continuada ya que, como escribe Porres ${ }^{25}$, el nieto de Garci Laso y de Sancha de Guzmán, «casado con doña Aldonza Niño de Guevara, fijó su residencia familiar en Cuerva». Con frecuencia permanecería ausente de su casa de San Román, lo que dejaba un tiempo suficiente para la transformación de su plaza en un muladar.

\section{Los bienes en el primer cuarto del siglo XVII}

En 1615, Pedro Laso Niño de la Vega, conde de $A \operatorname{Aros}^{26}$, biznieto del hermano mayor del poeta Garci Laso, pide al rey autorización para vender «las casas principales de mi mayorazgo con todas sus acesorias que son en la ciudad de Toledo a la parroquia de San Román». El rey contesta favorablemente y permite la venta de sus casas porque están «viejas y maltratadas» y porque tiene

\footnotetext{
24 Santa Clara,1599, fol. $10 \mathrm{v}, 11 \mathrm{r}$.

25 J. Porres Martin-Cleto, Historia de las calles de Toledo, 1988, t. II, p. 627.

26 J. PorRes (libro citado) recordó que don Garci Laso de la Vega fue nombrado primer conde de Arcos por el rey Felipe II (1558-1598).

Hispania Sacra, LX

121, enero-junio 2008, 131-142, ISSN: 0018-215-X
} 
«otras mejores a la parroquia de San Lorenzo» ${ }^{27}$. En el año 1616, el conde de Arcos vende sus bienes en la parroquia de San Román a la Compañía de Jesús. El documento de venta (Anejo 1), conservado en los archivos del Hospital de la Misericordia, indica la composición de estos bienes de la rama mayor del comendador Garci Laso de la Vega (Figura 3).

En 1616, las casas del conde de Arcos comprenden una casa principal junto a la calle Real, lindante con la casa de Antonio de Padilla y con la pequeña plaza de la casa, y siete casas accesorias. Una casa accesoria linda con la casa principal del conde de Arcos y la de Antonio de Padilla; otra abre a la entrada de la plazuela; debajo de ella está la cochera de la casa principal. Tres pequeñas casas accesorias caen dentro de la plazuela de la casa principal; dos pequeñas lindan con la placita y tienen su puerta a la calle que va de las Tendillas de Sancho Bienayas a la casa del secretario Vargas (actual calle de las Tendillas). Además, la propiedad del conde de Arcos incluye la placita a la cual abre una casa de la capilla de Cuerva ${ }^{28}$. Todos estos bienes están libres de tributo, menos el sitio donde se sitúa la caballeriza, dado con cargo al conde y a Antonio de Padilla por el Ayuntamiento en 157429.

Un año después, en 1617, el Hospital de la Misericordia recibe del Ayuntamiento un acuerdo favorable a su petición del cerramiento de la plazuela frontera de su casa, anteriormente del conde de Arcos, «dejando dentro de esta las dichas casas sin servicio de el dicho postigo...». En su petición recorda el hospital que su casa tiene una puerta principal que «sale a la calle donde vivían las descalzas...» y también «otro postigo que sale a la plazuela del conde de Arcos a las tendillas de Sancho Minaya por donde la dicha casa se servia...». Así sé acabara el uso de dicha plaza, mencionada por primera vez en 1438 en el testamento de Leonor de Toledo.

En el primer cuarto del siglo XVII, la casa del conde de Arcos se sitúa entre la casa de Antonio de Padilla al Oeste, la calle real al Sur, varias casas privadas sobre la calle que va de las Tendillas a la casa del secretario Vargas al Nor-Este, y un callejón al Norte. No linda con la plaza de Padilla, pero incluye una plazuela que comunica con la calle de las Tendillas por medio de una calle, la cual, según las observaciones de sus restos, está en codo, y de un callejón corto. La subida del callejón de las Tendillas hacia la plazuela se facilita por un pretil sobre el cual se pone un postigo en 1591.

\footnotetext{
27 A. D. P. T. Misericordia, Leg. 2, $n^{\circ}$ 9, 1504-1616.

${ }^{28}$ Doña Isabel de Desa fundó «una capellanía que dotó entre otras casas de una ... a la parroquia de santa Leocadia en una plaçuela frontero de las casas que heran del conde de arcos...» En 1617, el Hospital de la Misericordia pagara 2000 ducados por cerrar «el paso a unas casas que eran propiedad de la capellanía fundada en Cuerva por Doña Isabel de Saha», Leg. $2^{\circ}, \mathrm{n}^{\circ} 6$.

${ }^{29}$ «con cargo de tres mil quinientos maravedis de censo e decima que paga el dicho señor conde los dos mil docientos maravedis...», A. D. P. T. Misericordia, Leg. 2, n 9, fol. 35 v.
} 
La cochera de la casa de los Laso de la Vega se sitúa sobre la calle que sube de las Tendillas hacia San Román (actual calle de Esteban Illán), ocupa probablemente el lugar de la casa bodega de doña Leonor de Toledo, citada en 1438. Formulamos la hipótesis de que la cochera corresponde a la portada registrada en la primera mitad del siglo XIX ${ }^{30}$ y en siglo XX. Se trata de una puerta de tipo mudéjar fechable hacia finales del siglo XV.

\section{CONCLUSIÓN}

1. El análisis de los documentos de los siglos XV, XVI y XVII reunidos en los archivos del Hospital de la Misericordia conduce a poner de manifiesto la existencia de una plazuela, al Norte, delante de las casas principales que fueron sucesivamente de doña Leonor de Toledo, del obispo don Vasco Ramírez de Rivera, de don Garci Laso de la Vega y doña Sáncha de Guzmán. Más tarde, probablemente en la segunda mitad del siglo XVI, dicha plazuela se privatiza. De la calle que baja de las Tendillas de Sancho Bienayas se accede a la plazuela por una calle en codo o por un callejón. En cualquier caso, un pretil resuelve la fuerte pendiente debido a una desnivelación de unos nueve metros. A partir de 1617, el Hospital de la Misericordia, transformando la casa para su nueva función, llega a cerrar la plazuela y el pretil ${ }^{31}$. Cerrada la plazuela, esta será olvidada, lo que explica la confusión constante entre dicha plazuela y la plaza de Padilla, en la cual numerosos autores ven el limite Oeste de la casa de la familia de los Laso de la Vega ${ }^{32}$.

2. El estudio de las casas que ocupan la colina de San Román, que hemos podido visitar ${ }^{33}$, refuerza la información extraída de los documentos. Una vez situada la plazuela de la casa principal, hemos intentado ver lo que queda de ella dentro del espacio atribuido al hospital: no queda de ella, en el siglo XX, más que un pequeño espacio triangular. Pero el callejón frente a la casa principal que bajaba a la calle de las Tendillas apareció en la rehabilitación de la casa $\mathrm{n}^{\circ} 15$ de la calle de las Tendillas; fue despejado, limpiado y mantenido sin techo dentro de la casa nueva. También se puso a la vista el pretil y el muro de con-

${ }^{30}$ En 1840, esta puerta es de la casa ${ }^{\circ} 6$ calle de la Misericordia (actual calle Esteban Illán), propiedad del Marqués de Hermosillo (AMT, Padrón 1840). El pintor Arredondo realizó un cuadro titulado «Palacio de Hermosillo», conocido por una fotografía hecha por C. Alguacil. Se reconocen en esta fotografía los elementos recogidos y descritos por Téllez en su artículo «Estilo mudéjar toledano», BRABAC, Toledo, 1946, lam. 2, y 3 .

${ }^{31}$ A. M. T., 1257, Hospitales: «...en nombre del hospital... pedimos que a mayor abundamiento de licencia al dicho hospital, para que el pretil de la dicha plazuela se cierre, y se continue la fabrica y labor, que tiene ordenada...»

32 A. H. P. T., Toledo, J. M. Rojas, 1997, ya citado.

${ }^{33}$ Agradezco todas las personas que me han ayudado en estas investigaciones.

Hispania Sacra, LX

121, enero-junio 2008, 131-142, ISSN: 0018-215-X 
tención de la plazuela, hecho de un aparejo mudéjar de piedra e hiladas de ladrillos. La calle en codo y un pretil todavía se pueden apreciar desde la parte norte de la Universidad (Figura 4).

3. Por lo que se refiere a la casa del poeta, nunca ha estado en la calle a la cual se le ha dado su nombre ${ }^{34}$, ni en la casa ${ }^{\circ} 1$ del antiguo cobertizo de Santo Domingo el Antiguo. En este lugar, sobre las seis casas pequeñas tributarias al monasterio de Santa Domingo el Antiguo 35 notadas en 1572, dicho monasterio hizo construir, en $1580^{36}$, por el arquitecto Nicolás de Vergara el mozo, una casa de estilo renacentista, en ruinas en el último cuarto del siglo XIX.

4. Mientras, si consideramos que la fecha del nacimiento del poeta Garcilaso es el año $1498^{37}$, quizás poco antes, parece muy probable que el poeta nació a la casa principal comprada por el comendador Garci Laso. Ha podido vivir en esta casa (Figura 5), por lo menos de vez en cuando, hasta casarse en 1525. De esta casa, la fachada principal ha desaparecido desde 1617, pero el alineamiento de la pared sobre la calle que sube a San Román sigue siendo el mismo.

ANEXo

A. D. P. T., Misericordia Leg. 2, n 9, fol. 29 v. 1615-1616:

«... de casas principales que tiene en San Roman... alinda cassas de Antonio Padilla y por otra la plazuela de las dichas casas por delante con las calle Reales... de una casa açesoria a las dichas principales que alindan con ella por una parte y por otra parte con casa de Antonio Padilla. Yten otra casa açesoria que esta a la entrada de la plazuela de las dichas casas principales que tiene la puerta dentro de ella y esta debajo de la cochera de las dichas casas principales. Yten otras tres casas pequeñas açesorias que caen dentro de la dicha plazuela de las dichas casas principales... Yten otras tres casas açesoras que caen a la calle real que va de las tendillas de Sancho Bienayas a la casa del secretario Bargas que alinda una con otra, que por las espaldas linda con la plazuela de las dichas principales... Yten la plazuela que tiene las dichas casas principales según y como el dicho conde las a tenida y tiene y la pertenecen a la qual dicha plaçuela tiene un postigo una casa de capilania que fundo doña Ysabel a las ques patron del dicho señor conde Arcos... todas las cuales dichas casas principales y açesorias y el uso de la dicha plazuela asi como las agora y tiene regido el señor conde son... libres de todo tributo perpetuo... ecepto que sobre un sitio que dio la ciudad al dicho conde y al dicho antonio de padilla que junta es en que esta caballeriza y encima unas paneras les dio a cargo 3500 maravedis de censo a decima...»

\footnotetext{
${ }^{34}$ Esto se debe, sin duda, a una interpretación aproximativa del documento S. D. R., 517, ver nota 21 de este artículo.

35 A. S. D. A., Leg. 5, doc. 9, 1572 ;

${ }^{36}$ A. S. D. A. Plano y elevación.

37 J. L. PÉREz López, La fecha de nacimiento de Garcilaso de la Vega a la luz de un documento biográfico, Criticón, Toulouse, 78, 2000, p. 45-57.
} 
Figura 1. Plano esquemático de las casas de doña Leonor de Toledo y de sus linderos en 1438 .
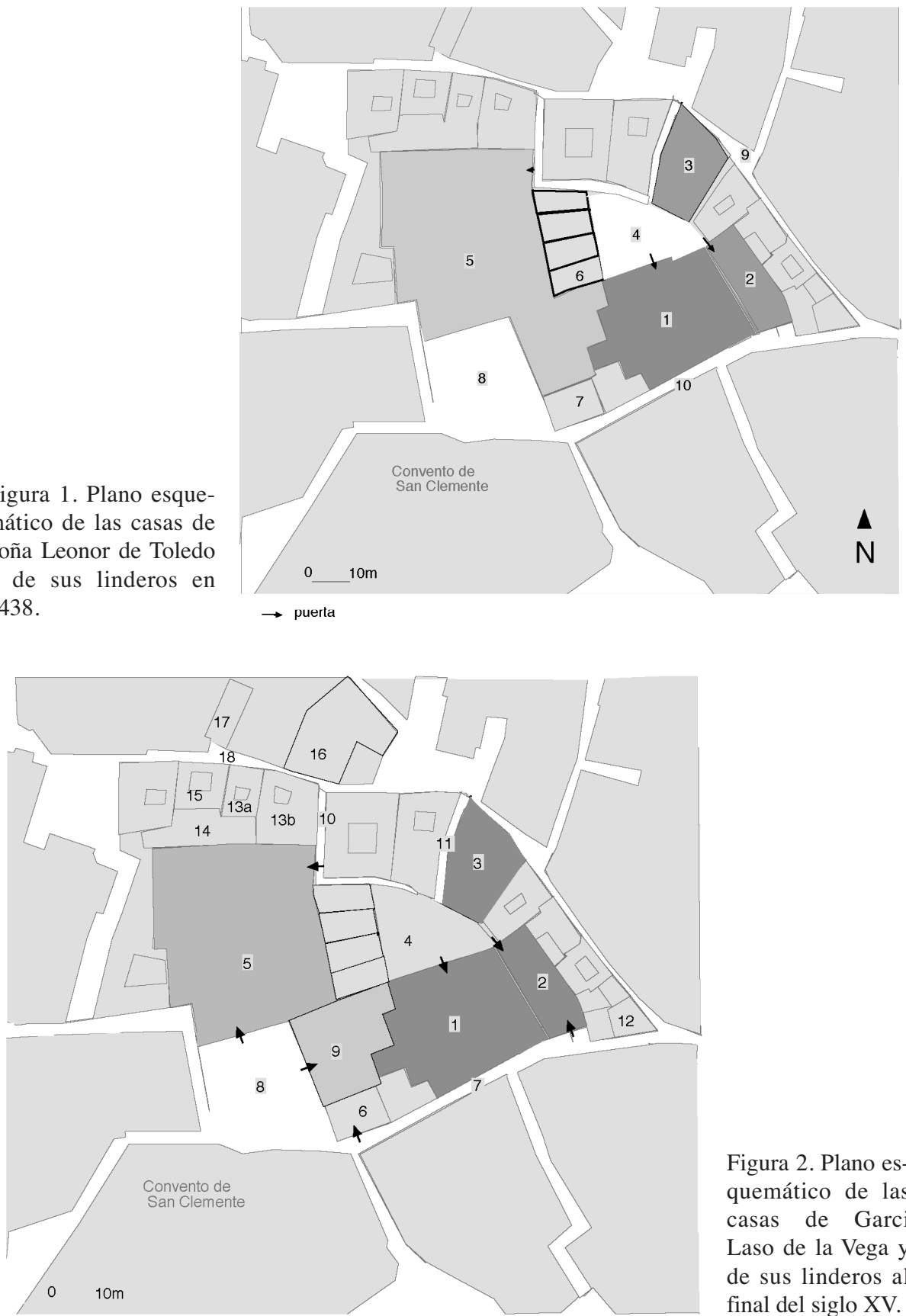

Figura 2. Plano esquemático de las casas de Garci Laso de la Vega y de sus linderos al final del siglo XV.

Hispania Sacra, LX

121, enero-junio 2008, 131-142, ISSN: 0018-215-X 


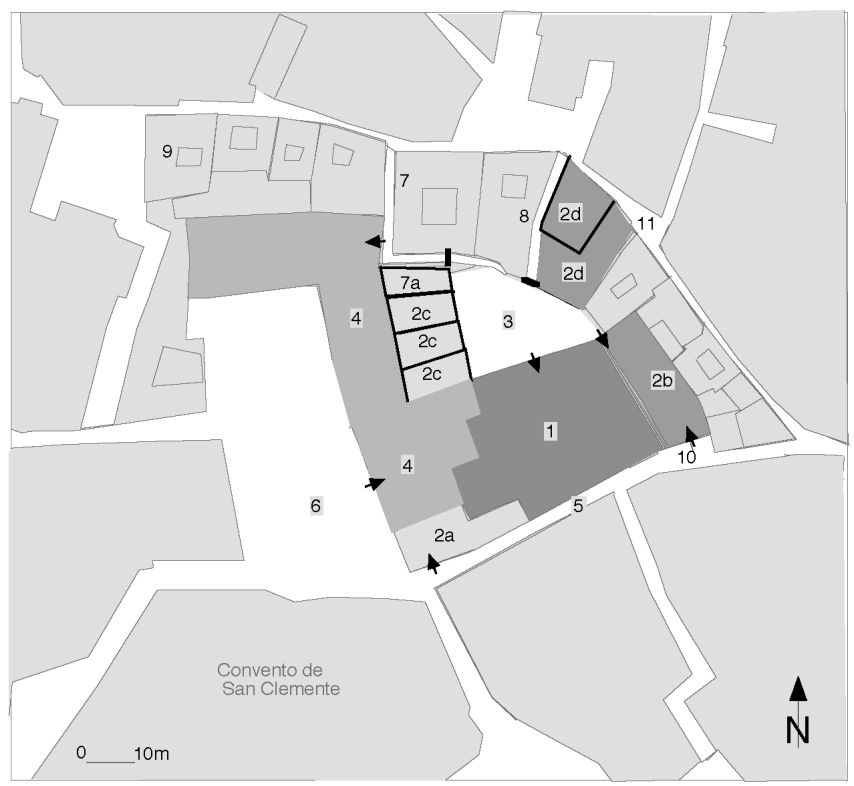

- postigo

$\rightarrow$ Puerta de entrada
Figura 3. Plano esquemático de las casas de Pedro Laso de la Vega Niño y Guzmán, conde de Arcos y de sus linderos en 1615.

Figura 4. Rinconada del callejón que abría antes a la calle de San Idelfonso (vista desde la Universidad de Castilla-La Mancha).

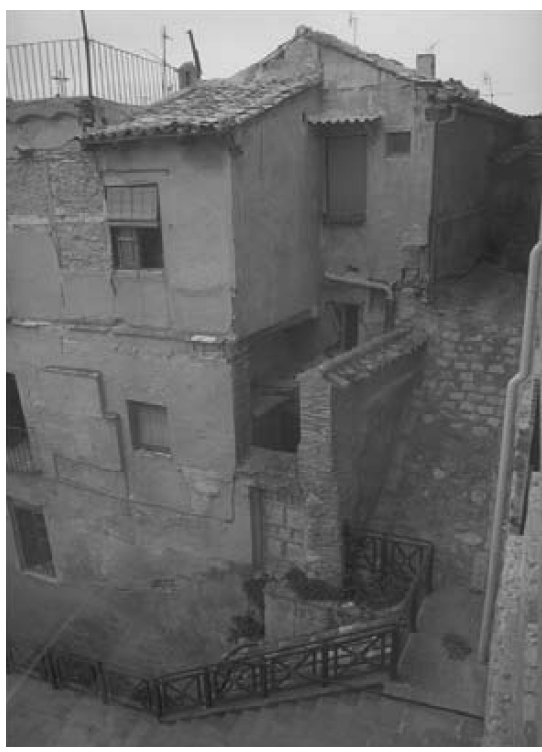

Hispania Sacra, LX 121, enero-junio 2008, 131-142, ISSN: 0018-215-X 


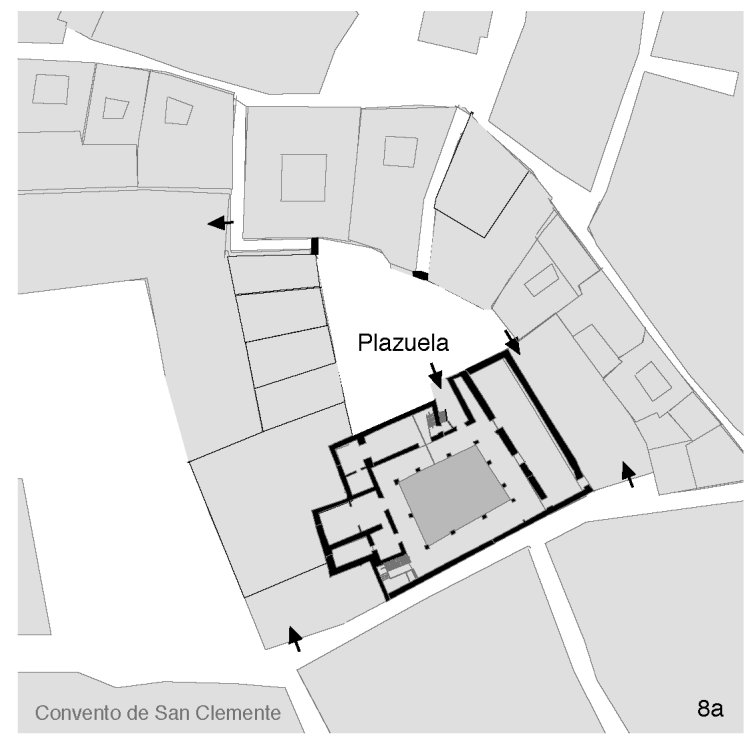

$$
\longrightarrow \text { puerta }
$$

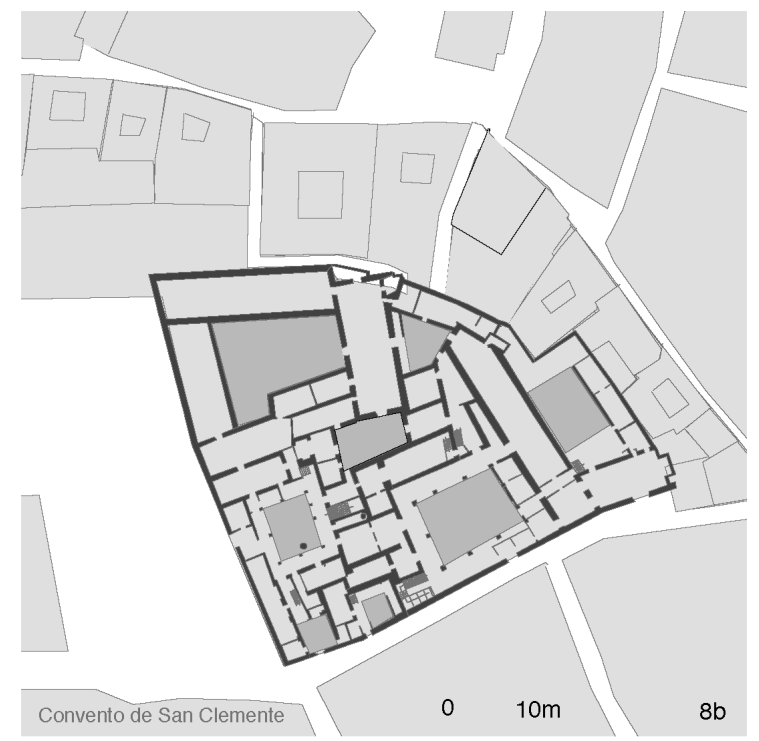

Figura 5. Restitución hipotética del plano de la casa principal de Garci Laso de la Vega en el siglo XV (8 a) a partir del plano del Hospital de la Misericordia en el siglo $\mathrm{XIX}^{38}(8 \mathrm{~b})$.

${ }^{38}$ I. G. N., 1881.

Hispania Sacra, LX

121, enero-junio 2008, 131-142, ISSN: 0018-215-X 